\title{
Health and Growth of Veal Calves Fed Milk Replacers With or Without Probiotics
}

\author{
H. M. Timmerman, ${ }^{1}$ L. Mulder, ${ }^{2}$ H. Everts, ${ }^{1}$ D. C. van Espen, ${ }^{3}$ E. van der Wal, ${ }^{3}$ G. Klaassen, ${ }^{4}$ \\ S. M. G. Rouwers, ${ }^{4}$ R. Hartemink, ${ }^{5}$ F. M. Rombouts, ${ }^{5}$ and A. C. Beynen ${ }^{1}$ \\ ${ }^{1}$ Department of Nutrition, Faculty of Veterinary Medicine, Utrecht University, 3508 TD Utrecht, The Netherlands \\ ${ }^{2}$ Research and Development Department, Winclove Bio Industries B.V. Amsterdam, The Netherlands \\ ${ }^{3}$ Research and Development Department, VanDrie Group, Mijdrecht, The Netherlands \\ ${ }^{4}$ Research and Development Department, Sloten B.V., Deventer, The Netherlands \\ ${ }^{5}$ Laboratory of Food Microbiology, Department of Agrotechnology and Food Sciences, \\ Wageningen University, Wageningen, The Netherlands
}

\section{ABSTRACT}

Four experiments with 1-wk-old veal calves were conducted to assess the influence of probiotics on growth and health indicators. In experiments 1 and 2 , the liquid probiotic supplements were administered daily from experimental $d 1$ to 15 . The treatment period in experiments 3 and 4 was extended to $56 \mathrm{~d}$. The probiotics used were a multispecies probiotic (MSPB) containing different probiotic species of human origin, or a calfspecific probiotic (CSPB) containing 6 Lactobacillus species isolated from calf feces and selected on the basis of a combination of characteristics.

When the data for the 4 experiments were pooled, the probiotics enhanced growth rate during the first 2 wk. During the 8-wk experimental period, average daily gain and feed efficiency were significantly improved in the probiotic-treated groups. The MSPB-induced increase in weight gain was greater when the control calves were considered less healthy based on a health score (an index of diarrhea and therapeutic treatments). Probiotic treatment tended to diminish mortality. The CSPB treatment reduced the incidence of diarrhea and the fecal counts of coliforms. When therapeutic treatment was intensive in the control calves, the ingestion of probiotics reduced the percentage of calves that required therapy and the amount of treatments needed against digestive or respiratory diseases. There was no clear difference in the efficiency of the MSPB and CSPB preparations. Further research is necessary to identify underlying mechanisms and to evaluate the potential of probiotics to improve respiratory health in veal calf production.

(Key words: veal calves, probiotics, growth performance, animal health)

Received August 14, 2004.

Accepted January 25, 2005.

Corresponding author: H. Timmerman; e-mail: h.timmerman@ vet.uu.nl.
Abbreviation key: ADG = average daily gain, $\mathbf{C S P B}=$ calf-specific probiotic, $\mathbf{F E}=$ feed efficiency, $\mathbf{G H S}=$ general health score, LAMVAB = Lactobacillus anaerobic MRS agar with vancomycin and bromocresol green, MRS = de Man, Rogosa, and Sharpe, MSPB = multispecies probiotic.

\section{INTRODUCTION}

Under current husbandry conditions, veal calves are often affected by diarrhea and respiratory disease. Diarrhea is the main cause of morbidity and mortality in the early life of veal calves, and the first peak of respiratory diseases often emerges at $4 \mathrm{wk}$ of age, causing substantial economic losses due to medication and growth depression (Postema et al., 1987).

Various factors could cause the high incidence of intestinal and respiratory disease in veal calves. After birth, calves are separated from their mothers, preventing the calf from picking up the protective gut flora from its mother (Fuller, 1989). Furthermore, at a very young age, the animals are faced with major stress events like transportation, marketing, dietary changes, and exposure to a variety of infectious agents. Consequently, animals consume less milk (Loerch and Fluharty, 1999), are predisposed to loss of barrier function of the gut (Nabuurs et al., 2001; Soderholm and Perdue, 2001), and may suffer from impaired immune function (Blecha et al., 1984; Sheridan et al., 1994). Moreover, the protective potential of the microbial gut flora tends to decrease (Cray et al., 1998). For example, during stress events, the trend is for the protective lactobacilli to decrease and for coliforms to increase (Fuller, 1989). To prevent the opportunistic pathogenic flora from flourishing, current practice (in the Netherlands) is to treat calves with prophylactic antibiotics during the first 5 to $10 \mathrm{~d}$ after arrival. However, the antibiotics diminish not only the activity of the pathogenic flora, but also that of the protective flora. 
To establish a protective flora in veal calves, the use of probiotics is promising. Various papers have addressed the antidiarrheal capacities of different probiotic strains in calves (Abe et al., 1995; Donovan et al., 2002; Khuntia and Chaudhary, 2002). Apart from their positive effects on gastrointestinal infections, probiotics may be used to prevent nonintestinal infectious conditions, such as respiratory tract infections (Hatakka et al., 2001). So far, the effect of probiotics, if any, on respiratory health in veal calves has not been described.

To further qualify the health improving capacity of probiotics in young veal calves, the effect of different kinds of probiotics on health and growth variables was investigated. Different probiotic concepts were tested, namely a multispecies probiotic (MSPB) and a calfspecific multistrain probiotic (CSPB). The MSPB was comprised of 6 probiotic strains of various genera. It was reasoned (Timmerman et al., 2004) that the combination of genera-specific probiotic properties should make the MSPB preparation superior to the traditional monostrain probiotics. The CSPB preparation used in this study contained 6 Lactobacillus strains that were originally isolated from the target animal. It was hypothesized that its calf specificity would enhance the ability of the CSPB to colonize the host animal. Because successful colonization is one of the prerequisites for a probiotic to exert its beneficial activity, it could be suggested that the CSPB should have greater effects than the MSPB. Furthermore, it should be noted that fresh fermented probiotic cultures were used instead of the usual (and more expensive) freeze-dried preparations.

Four experiments are described in this paper, conducted at 2 research facilities. In the first 2 experiments, only the MSPB preparation was tested. The probiotics were administered during the first $14 \mathrm{~d}$ after arrival of the calves. In the last 2 experiments, both the MSPB and CSPB preparations were tested, and the period of probiotic administration was extended to $8 \mathrm{wk}$.

\section{MATERIALS AND METHODS}

\section{Probiotic Strains}

Two liquid probiotic formulas were developed. The MSPB preparation contained commercially available probiotic strains; the CSPB formula contained strains isolated from veal calf digesta and feces. Freeze-dried probiotic strains of human origin were used for formulation of the liquid MSPB supplement. A combination of 6 strains was used: Lactobacillus acidophilus W55, Lactobacillus salivarius W57, Lactobacillus paracasei spp. paracasei W56, Lactobacillus plantarum W59, Lactococcus lactis W58, and Enterococcus faecium W54. All strains were obtained from Winclove Bio Industries
B.V. (Amsterdam, the Netherlands). To formulate the CSPB supplement, Lactobacillus strains were isolated from fresh fecal samples from healthy cattle and screened for probiotic properties.

\section{Isolation and Screening of Lactobacillus Strains from Veal Calves}

Fecal samples were collected freshly from cattle and stored in sterile buffered peptone water (Oxoid, Haarlem, the Netherlands). The samples were kept refrigerated and were processed on the same day. The fecal samples were homogenized in buffered peptone water with an Ultra Turrax blender (Janke and Kunkel, Staufen, Germany) under anaerobic conditions. Further processing was performed under aerobic conditions. Serial dilutions of the homogenized samples were made in reduced physiological salt solution $(1 \mathrm{~g} / \mathrm{L}$ neutralized bacteriological peptone, Oxoid; $0.5 \mathrm{~g} / \mathrm{L}$ L-cysteine-HCl, Sigma-Aldrich Chemie, Steinheim, Germany; and $8 \mathrm{~g} / \mathrm{L} \mathrm{NaCl}$, Acros Organics, Geel, Belgium), and cultured on Lactobacillus anaerobic MRS agar (Merck, Darmstadt, Germany) with vancomycin and bromocresol green (LAMVAB). This medium contained $52.2 \mathrm{~g} /$ L MRS broth (Merck), $0.25 \mathrm{~g} / \mathrm{L}$ cysteine-HCL, $0.025 \mathrm{~g} /$ $\mathrm{L}$ bromocresol green (Sigma-Aldrich), $20 \mathrm{~g} / \mathrm{L}$ agar and was supplemented with $20 \mathrm{mg} / \mathrm{L}$ vancomycin hydrochloride (Sigma-Aldrich; potency $1000 \mu \mathrm{g} / \mathrm{g}$ ) (Hartemink et al., 1997). The plates were incubated anaerobically (Anoxomat, Mart, Lichtenvoorde, the Netherlands) at $37^{\circ} \mathrm{C}$ for $48 \mathrm{~h}$.

Isolated colonies with different appearances were picked from each plate, transferred to new LAMVAB plates, and incubated at $37^{\circ} \mathrm{C}$ for $48 \mathrm{~h}$ (anaerobic) to obtain pure strains. Finally, 83 pure colonies were isolated and grown in MRS broth (Merck) at $37^{\circ} \mathrm{C}$ for 24 h. These strains were screened for useful properties to produce a liquid probiotic supplement. The cultures in MRS broth were stored in $30 \%$ glycerol at $-80^{\circ} \mathrm{C}$. The 83 isolates were subjected to 7 tests, which are described in Table 1. A few strains that did not grow under aerobic conditions were eliminated. Twelve strains were selected based on data regarding growth rate, acidification rate, and inhibition of pathogens. The selected strains were then checked for growth and stability, as assessed by viable cell count after 2 wk of refrigerated storage at $4^{\circ} \mathrm{C}$, in a liquid fermentation medium (see below). Based on these results, 6 Lactobacillus strains were selected for identification by fermentation patterns with standard analytical profile index (API 50CHL, BioMérieux, Inc., Hazelwood, MO) tests. The selected isolates with their identification and screening results are presented in Table 1. 


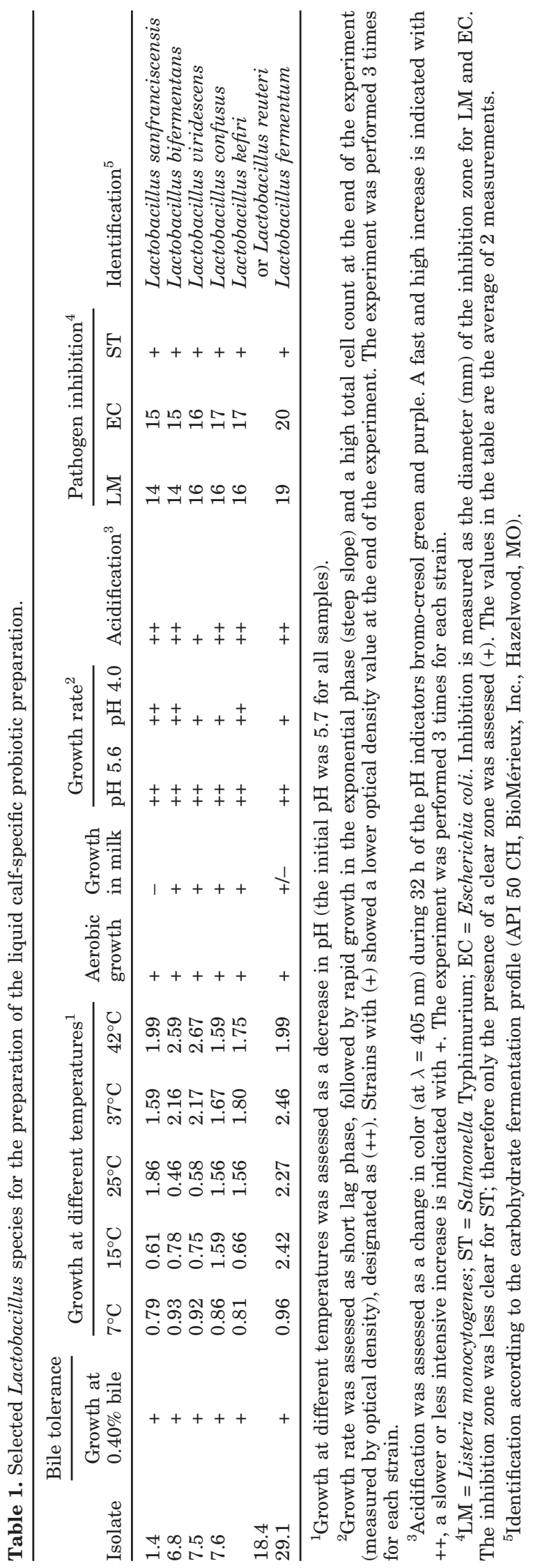

\section{Application of MSPB and CSPB Preparations}

For this study, a new probiotic application, called Silogic, was developed. This liquid growth medium was composed of soy protein hydrolysate (Soyprotein Hydrolysate Powder Pure, Heybroek, the Netherlands), yeast extract (Gistex LS powder AGGL, Gistbrocades, the Netherlands), dextrose (Amylum Europe, Aalst, Belgium), and minerals (a combination of potassium chloride, magnesium sulfate, and manganese sulfate; Kortex, Orsingen-Nenzingen, Germany). These ingredients $(16 \mathrm{~g} / \mathrm{L})$ were dissolved in $25 \mathrm{~L}$ of hot tap water. Then, $25 \mathrm{~L}$ of cold tap water was added. The product was quickly cooled and stored refrigerated $\left(4^{\circ} \mathrm{C}\right)$ in small containers. For formulation of the MSPB and CSPB preparations, the component strains (pregrown in MRS broth and stored at $-80^{\circ} \mathrm{C}$ ) were first individually inoculated at $10 \mathrm{~mL} / \mathrm{L}$ and grown overnight, at $37^{\circ} \mathrm{C}$ in a liquid fermentation medium.

After fermentation, the $\mathrm{pH}$ of the cultures was determined $(\mathrm{pH}<4.2)$, and the optical density of the cultures was measured using a spectrophotometer $(\lambda=620 \mathrm{~nm})$ to assure growth of all strains (optical density $>1.000$ ). Samples were taken for viable cell count analysis of each strain. Then, the cultures were mixed (in equal volumes) and a sample was taken to determine the total viable cell count of the finished product $\left(\sim 10^{9} \mathrm{cfu} / \mathrm{mL}\right)$. The product was kept refrigerated at $4^{\circ} \mathrm{C}$ and was stored for up to $2 \mathrm{wk}$. During storage, the total cell count was checked weekly to control stability of the product.

\section{Experimental Setup}

A short summary of all 4 feeding experiments is given in Table 2.

Experiment 1. Experiment 1 took place at the research station of the VanDrie Group. The animals were reared according to the all-in/all-out management system, meaning that animals of similar age enter and leave the facility at the same time.

Three hundred sixty male Holstein-Friesian calves, about $10 \mathrm{~d}$ of age, were purchased at a local market. The calves were housed individually in wooden stalls $(70 \times 170 \mathrm{~cm})$ with slatted floors. The animals were housed in 10 rooms (36 animals/room). Shortly after arrival, the animals were randomly allocated to 1 of 6 feed treatments based on their origin and BW. The treatments consisted of a commercial starter diet (Navobi Rood 3, Navobi member of the VanDrie Group, Staverden, the Netherlands), but with varying protein sources. All milk replacers were based on defatted milk powder, whey powder, lard, tallow, and coconut oil, and were formulated to contain $22 \% \mathrm{CP}$ and $20 \%$ fat. The milk replacers were reconstituted in hot water $\left(65^{\circ} \mathrm{C}\right)$ and fed at a temperature of approximately $41^{\circ} \mathrm{C}$. On 


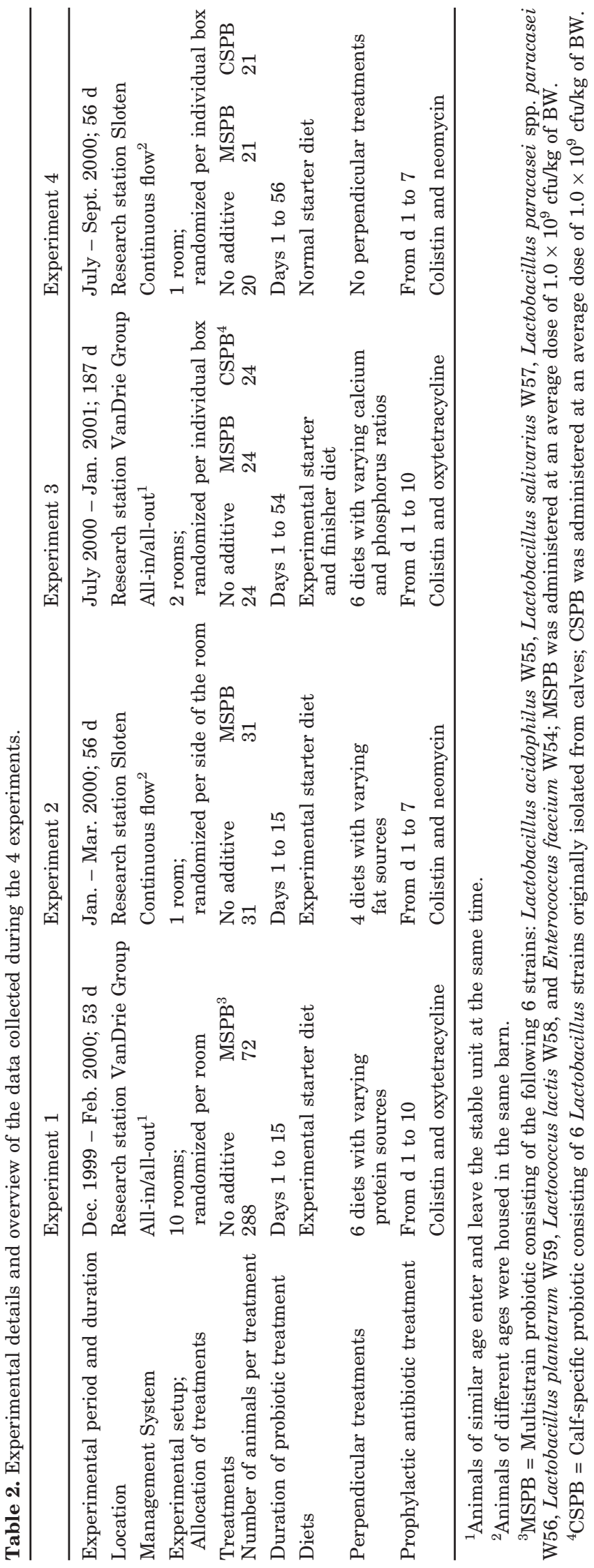

the day of arrival, the animals were fed twice with $2 \mathrm{~L}$ of an electrolyte solution $(20 \mathrm{~g} / \mathrm{L}$ water, Elektrolytenmix, Navobi). One day after arrival, the animals received $1.7 \mathrm{~L}$ containing $220 \mathrm{~g}$ of air-dry milk replacer per meal, the volume being gradually increased to $6.4 \mathrm{~L}$ containing $820 \mathrm{~g}$ of air-dry milk replacer per meal after 8 wk. The calves were fed twice a day, at 0300 and 1500 $\mathrm{h}$, with one of the reconstituted milk replacers presented in plastic buckets. Of 360 calves, 72 calves received the MSPB preparation and the other calves served as controls. Among the calves that were given MSPB and the control calves, all 6 dietary treatments mentioned above were equally distributed. The 72 calves were treated with MSPB from d 1 to 14 after arrival. All animals received $45 \mathrm{~mL}$ (an average dose of $1.0 \times 10^{9} \mathrm{cfu} / \mathrm{kg}$ of BW) of the probiotic mixture with the afternoon feeding. The MSPB preparation was put into the bucket immediately before the milk was added. All calves were treated with colistin sulfate $(4 \%, 2 \mathrm{~g} / \mathrm{d}$ per calf, Dopharma, Raamsdonksveer, the Netherlands) and oxytetracycline-HCl (2 g/d per calf, Dopharma) during the first $10 \mathrm{~d}$. This antibiotic mixture was combined with the morning feeding.

Experiment 2. Experiment 2 took place at the research station of Sloten B.V. The animals were housed according to a continuous management system. This means that animals of different ages were present in the same facility, albeit in different rooms. The same MSPB preparation was tested as in experiment 1 . All experimental calves were kept in one unit. The calves were housed in wooden stalls as described above. Sixtytwo male Holstein-Friesian calves, at about $10 \mathrm{~d}$ of age, were purchased at a local market. They were split into 2 experimental groups, the groups being housed at opposite sides of the unit. The animals were allocated to 1 of the 2 groups so that the distributions of origin and BW were similar between groups. Three dietary treatments were randomly and equally distributed over the control and test calves. Treatments comprised a normal starter diet (Spraymes Start, Sloten B.V.) with varying sources of fat. All milk replacers were based on whey powder, delactosed whey powder, lard, fish oil, and coconut oil, and were formulated to contain $22.5 \%$ $\mathrm{CP}$ and $16.5 \%$ fat. On the day of arrival, animals were fed twice with $2 \mathrm{~L}$ of an electrolyte solution $(10 \mathrm{~g} / \mathrm{L}$ water, Emix, Sloten). One day after arrival, the animals received $1.5 \mathrm{~L}$ containing $150 \mathrm{~g}$ of air-dry milk replacer per meal, the volume being gradually increased to 6.0 $\mathrm{L}$ per meal containing $725 \mathrm{~g}$ after $8 \mathrm{wk}$. Thirty-one calves were treated with MSPB from d 1 to 14 after arrival. During this period, animals were fed 3 times a day (at 0700,1230 , and $1800 \mathrm{~h}$ ). With the morning feeding, MSPB was supplied at an average dose of 1.0 $\times 10^{9} \mathrm{cfu} / \mathrm{kg}$ of BW. The noon feeding consisted of luke- 
warm water with an electrolyte solution and antibiotics. The antibiotics (colistin and neomycin) were also added to the afternoon feeding. Antibiotic treatment started $1 \mathrm{~d}$ after arrival and continued for the 7 subsequent days (colistin sulfate 4\%; $2.0 \mathrm{~g} / \mathrm{d}$ per calf, Dopharma). Neomycin sulfate $(0.7 \mathrm{~g} / \mathrm{d}$ per calf equivalent to $0.49 \mathrm{~g}$ of pure neomycin, Dopharma) was only administered during the first $5 \mathrm{~d}$ of the experiment.

Experiment 3. Experiment 3 was conducted at the same research station as experiment 1 . After carrying out the first 2 experiments, the CSPB preparation had been developed. The 2 mixtures were administered for an 8-wk period (normally referred to as the starter phase) at an approximate dose of $1.0 \times 10^{9} \mathrm{cfu} / \mathrm{kg}$ of BW. During the first $2 \mathrm{wk}, 45 \mathrm{~mL}$ of MSPB or CSPB probiotic $\left(1.0 \times 10^{9} \mathrm{cfu} / \mathrm{mL}\right)$ was administered and during wk 3 to 4,5 to 6 , and 7 to 8 , the daily dose was 50 , 60 , and $80 \mathrm{~mL}$, respectively.

Two stable units comprising 72 animals were used. The animals were assigned based on weight and origin to a control group, a group receiving MSPB, and a group receiving CSPB. The 3 groups were equally distributed across the 2 units. Evenly distributed over the 3 treatment groups were 6 dietary treatments consisting of various Ca:P ratios. Feed characteristics and the feeding regimen were similar to that used in experiment 1.

The animals were followed for the entire fattening period ( 8 to 26 wk of age) so that any carryover effects of probiotic treatment beyond the first 8 wk could be monitored.

Experiment 4. This experiment was conducted at the same research station as described for experiment 2 . The design of the experiment, i.e., probiotic treatment and allocation of treatments were similar to those described for experiment 3 . Feed characteristics and feeding regimen were similar to that used in experiment 1 , except that probiotic treatment was the only variable tested.

\section{Fecal Collection and Enumeration of Lactobacilli and Coliforms}

On d 5, 12, and 50 of experiments 3 and 4, fecal samples were collected from 18 randomly selected animals so that there were 6 samples per treatment. Fecal samples were collected fresh from calves upon rectal stimulation and were stored in sterile buffered peptone water (Oxoid), enriched with $0.5 \mathrm{~g} / \mathrm{L}$ of L-cysteine- $\mathrm{HCl}$ (Sigma-Aldrich). The samples were kept refrigerated and processed immediately. The fecal samples were weighed and then homogenized in buffered peptone water using an Ultra-Turrax mixer under anaerobic conditions. Further processing was performed under aerobic conditions. Dilutions of the homogenized samples were made in reduced physiological salt solution (Oxoid).

Relevant dilutions were plated on LAMVAB (Hartemink et al., 1997) and eosin methylene blue (Oxoid) media, using a spiral plater (Eddy jet, Leerdam, the Netherlands), for the determination of the total cell count of Lactobacillus spp. and coliforms, respectively. The LAMVAB plates were incubated anaerobically (Anoxomat, Mart) at $37^{\circ} \mathrm{C}$ for $48 \mathrm{~h}$. Eosin methylene blue agar plates were incubated aerobically at $37^{\circ} \mathrm{C}$ for 24 h. After incubation, the agar plates were assessed for growth and colonies were counted. Using the relevant calculations for the spiral plater, the total cell counts of lactobacilli and coliforms per gram of fecal material were calculated.

\section{Data Collection}

Growth performance data are presented for various intervals after arrival. On arrival (d 0), the calves were 1 wk old. Week 1 of the experiment refers to the first $7 \mathrm{~d}$ after arrival. In experiments 1 and 2, probiotic treatment was during the first $2 \mathrm{wk}$ and only data from the so-called starter phase (first $8 \mathrm{wk}$ after arrival) were collected. In experiments 3 and 4, probiotic treatment was extended to $8 \mathrm{wk}$. In experiment 3 , data were collected until slaughter, i.e., BW, feed intake, and slaughter quality were recorded. In experiment 4 , the animals entered another experiment after $56 \mathrm{~d}$ and the further data are not presented.

Calves were weighed individually on arrival, and thereafter in wk 2, 4, and 8. Calves in experiment 1 were not weighed at $2 \mathrm{wk}$ of age. Body weight at $2 \mathrm{wk}$ was estimated as [(BW at wk $4-$ initial BW ) / 2] - 2.5 $\mathrm{kg}$. Milk replacer intake was recorded daily throughout the trial. From this daily intake of milk replacer, the intake of air-dry milk replacer was calculated as (milk replacer offered - milk refused) $\times$ inclusion rate of dryair milk replacer per liter of water. Body weight gain, average daily gain (ADG), DMI, and feed efficiency $(\mathbf{F E})$ were calculated.

During all 4 experiments, the occurrence of diarrhea was recorded daily during the first $14 \mathrm{~d}$. Recording was done per animal and by one individual, who was unaware of treatment modality in experiments 3 and 4 . In experiments 1 and 3 , abnormal stool consistency was recorded, irrespective of its occurrence form as specified below. In experiment 2 , fecal scores were given per day per experimental group. In experiment 4 , the feces of each calf was inspected daily and stool appearance was scored as described below.

Mild diarrhea, most probably from nutritional origin, would be present if calves were vivid and had yellow and mostly liquid feces. Severe diarrhea, most probably 
from infectious origin, was scored if calves were apathetic and feces had an unpleasant odor and was slimy, watery, green, or yellow with blood present. Where necessary, body temperature was assessed to confirm the visual observations made.

The incidence of various diseases was estimated from the number of antibiotic treatments assigned by farm personnel or the veterinarian against digestive, respiratory, or other diseases such as joint or umbilicus infections. Antibiotic use per animal was recorded during the complete starter phase ( 1 to $8 \mathrm{wk}$ ). Antibiotic treatments were classified as therapeutic treatments needed for gastrointestinal, respiratory, or other (not shown in the tables) disease. The sum of all therapeutic treatments was calculated. Mortality was recorded during the complete starter phase.

To monitor overall health in each treatment group in each experiment, a general health score (GHS) was designed. The GHS score was developed before data evaluation and statistical analysis. The incidence of diarrhea and therapeutic treatments for digestive, respiratory, or other diseases were weighted differently in the following formula: GHS per animal $=15-1 \times$ total number of diarrheic days (irrespective of its nature) $2 \times$ the number of individual therapeutic treatments for digestive diseases $-3 \times$ the number of individual therapeutic treatments for respiratory diseases $-2 \times$ the number of individual therapeutic treatments for infections other than digestive or respiratory $-2 \times$ the number of antibiotic treatments on a herd basis. The weighting factor of each abnormality was based on its assumed impact on health.

\section{Statistical Analyses}

The individual calf was considered the experimental unit. Data from calves that died were included in the data set and accounted for until date of death. Body weight gain, $\mathrm{ADG}, \mathrm{DMI}$, and $\mathrm{FE}$ were adjusted by $\mathrm{AN}$ OVA using initial BW as a covariate. Fecal bacterial counts were transformed $\left(\log _{10}\right)$ before statistical analysis. Means were calculated by the least significant difference method of SAS (SAS Institute, 2000). When significant differences $(P<0.05)$ due to probiotic treatment were detected, differences were evaluated with a Student's $t$-test using the GLM procedure of SAS (SAS Institute, 2000). Data regarding the number of days with diarrhea, the number of antibiotic treatments, and the GHS were found to be not normally distributed as based on the Shapiro-Wilk test. Furthermore, skewness and kurtosis of the rest value of the models were not between -2 and 2 . Therefore, the continuous data were first transformed to an ordinal scale. Data for diarrheic days and antibiotic treatments were classified as fol- lows: no event (0), incidental (1), or recurring ( $\geq 2$ ). Data for the GHS were transformed as follows: healthy (12 to 15), slight illness (6 to 9 and 9 to 12), moderate illness ( 0 to 3 and 3 to 6 ), and severe illness (-18 to 0 ). Differences between control and probiotic treatments were compared with a Proportional Odds model using the logistic procedure of SAS (McCullagh and Nelder, 1989). Mortality, diarrhea incidence, and percentage of animals needing therapeutic treatments were evaluated by means of a $\chi^{2}$ test. The applied perpendicular dietary treatments in experiments 1 to 3 were not taken into account in the statistical analysis because they were always evenly distributed over the calves fed the milk replacers without or with probiotics. Moreover, statistical analyses revealed no interaction effect between probiotic and dietary treatments.

In Table 3 and Figure 1, data from all experiments are pooled. To minimize the effect of the different number of calves present in the treatments groups, a weight factor was introduced. This weight was calculated as 25 , being the mean group size of the control calves in experiments 2,3 , and 4 , divided by the number of animals present in a treatment group. In this pooled analysis, treatment had only 2 levels, i.e., control and probiotic-treated, experiment (1 to 4) was included as a block effect, and initial BW was used as a covariate. Means were adjusted for the experiment and initial BW by covariance. The level of statistical significance was preset at $P<$ 0.05 .

\section{RESULTS}

\section{Mortality and Growth Performance During the Starter Phase}

Mortality and growth performance are presented in Table 3. There was no significant effect of probiotic treatment on mortality, growth, and FE (feed:gain) in experiment 1 .

In experiment 2, there was a lower ADG from 1 to 8 wk compared with experiment 1 . No significant difference occurred regarding mortality, although 4 animals in the control group and 1 animal in the MSPB group died. Treatment with MSPB significantly enhanced growth from 1 to 2 wk: BW gain was $46 \%$ higher than in the control animals. This growth-promoting effect of MSPB was still noticeable at wk 8, but it was not statistically significant.

No mortality occurred in experiment 3. Calves fed MSPB or CSPB numerically increased BW gain during the first $2 \mathrm{wk}$. The probiotic-induced increase of BW gain was not statistically significant, although a significant improvement of $\mathrm{FE}$ for 1 to 2 wk was observed (data not shown). No significant differences regarding $\mathrm{BW}$ gain and FE were seen for wk 1 to 8 . There was a 


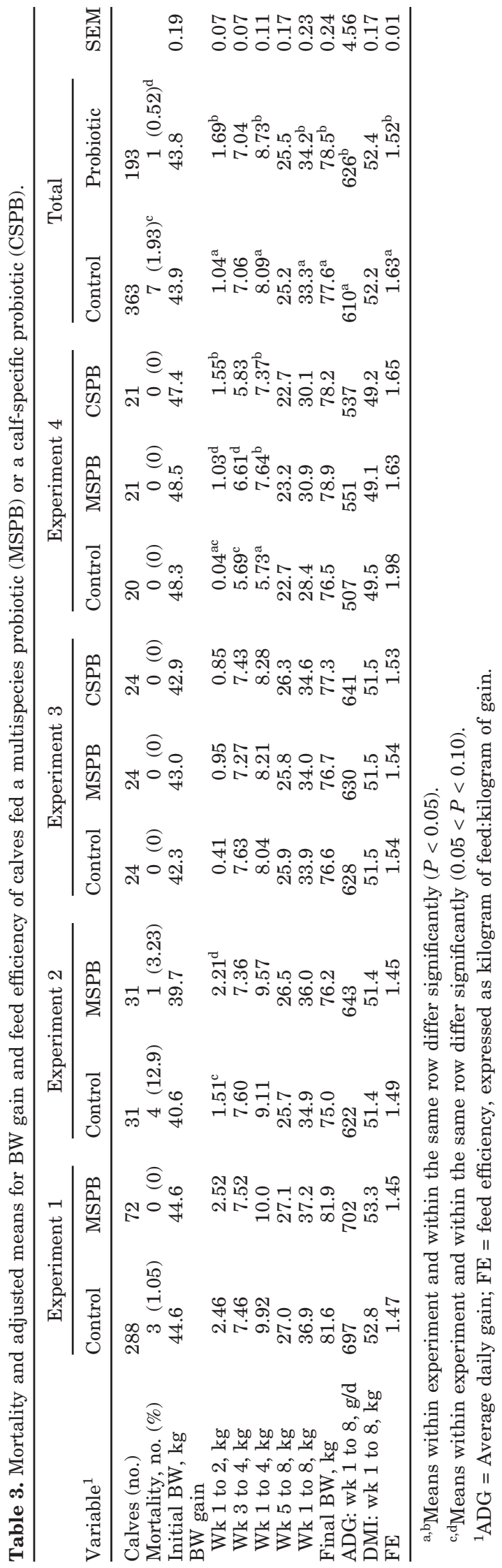

carryover effect of probiotic treatment in that it reduced digestion problems during the last phase of the 26 -wk fattening period and lowered the FE from wk 20 to 27 (data not shown). The result was a $4.0-$ and $4.1-\mathrm{kg}$ higher carcass weight for the CSPB- and the MSPBtreated animals, respectively. The increase in carcass weight did not reach statistical significance.

There was no mortality in experiment 4 . Body weight gain was low compared with that found in experiments 1,2 , and 3 . Both probiotic treatments markedly enhanced BW gain. The growth-promoting effect of CSPB was statistically significant for wk 1 to 2 and 1 to 4 , whereas MSPB treatment significantly increased BW gain for wk 3 to 4 and 1 to 4 . The growth-promoting effect was still present for wk 1 to 8 but it was not statistically significant. Feed efficiency over the 8-wk period was numerically decreased after treatment with MSPB or CSPB (-18\% and $-17 \%$; NS).

When all data were pooled, probiotic treatment was associated with a significantly enhanced growth during the first $2 \mathrm{wk}$ and in the periods 1 to 4 and 1 to $8 \mathrm{wk}$ of the starter phase. Body weight at 8 wk was significantly higher after probiotic treatment. Average daily gain and $\mathrm{FE}$ from wk 1 to 8 were significantly improved after feeding probiotics $(+2.6 \%$ and $-6.7 \%)$. Furthermore, probiotic treatment tended to lower mortality.

\section{Diarrhea Incidence and Duration}

Table 4 shows the percentage of animals suffering from diarrhea and the average diarrheic days per animal per treatment group. In experiment 2 , no individual data were collected so that the percentage of animals with diarrhea and the average duration could not be calculated. In experiment 2 and 4, a distinction was made between mild and severe diarrhea.

There was a low incidence of diarrhea in experiment 1 compared with the other experiments. Treatment with MSPB caused a reduction in average duration of diarrheic episodes, but the reduction was not statistically significant (data not shown). In experiment 2 , the number of days with mild diarrhea was high. Treatment with MSPB resulted in a nonsignificant increase. In experiment 3, treatment with either MSPB or CSPB reduced the incidence of diarrhea. The calf-specific probiotic, unlike MSPB, diminished the duration of diarrhea. In experiment 4, CSPB treatment successfully suppressed mild diarrhea compared with MSPBtreated and control calves. Administration of MSPB had no significant influence on mild diarrhea. Both MSPB and CSPB treatment lowered the incidence of severe diarrhea, but the effects did not reach statistical significance. Treatment with CSPB significantly low- 


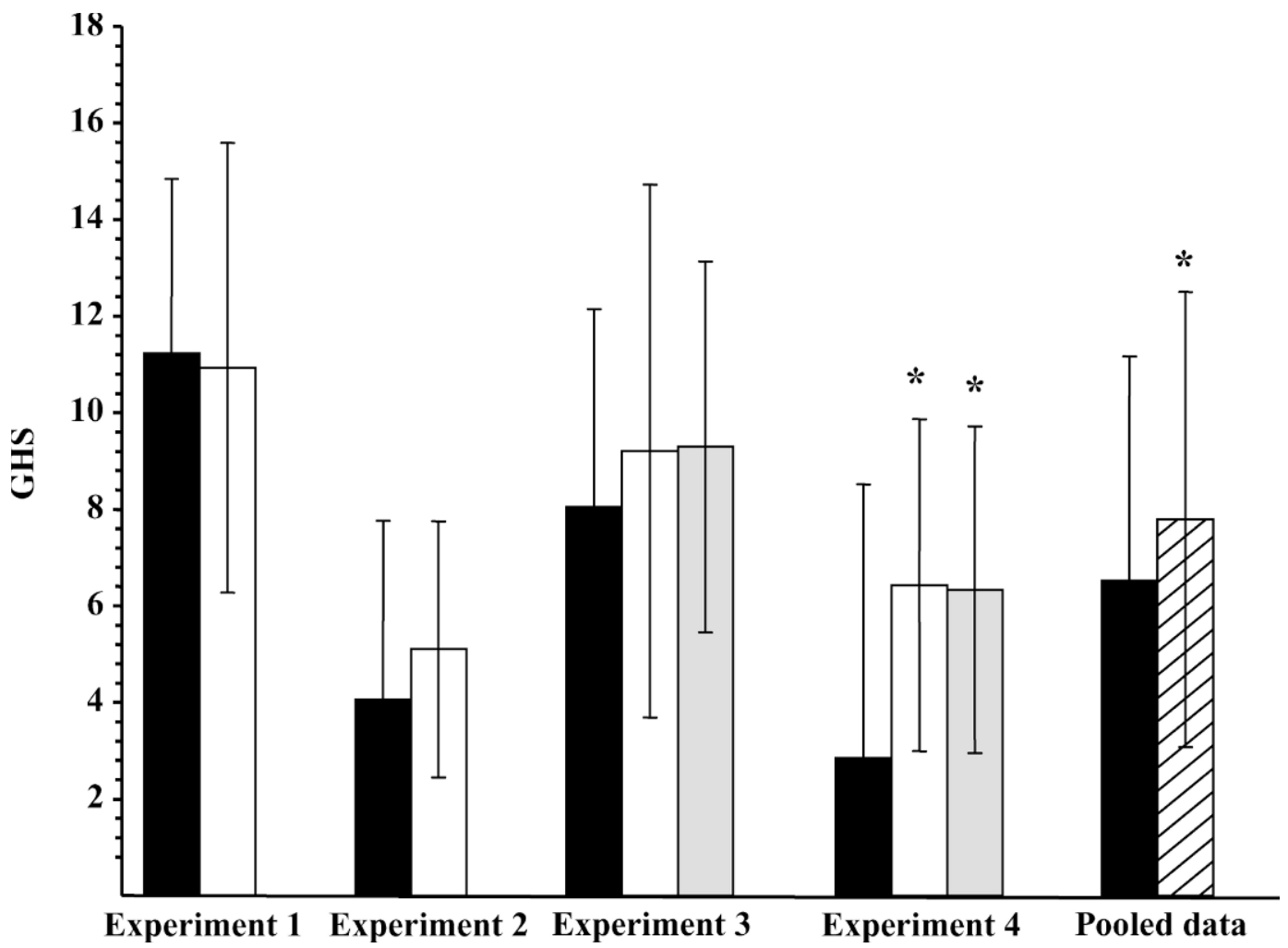

Figure 1. The general health score (GHS) of calves per experiment as affected by treatment with a multispecies probiotic (MSPB, white bars) or a calf-specific probiotic (CSPB, gray bars) vs. control treatment (black bars). Data from 4 experiments were pooled and had 2 levels, i.e., control and probiotic-treated (hatched bar) animals. *Significant differences $(P<0.05)$ with control treatment. Values are means \pm SD.

ered the incidence and duration of diarrhea $(-50 \%$ and $-58 \%$ ), irrespective of its nature.

\section{Fecal Coliform and Lactobacillus Counts in Experiments 3 and 4}

In experiments 3 and 4 , fecal samples were taken on d 5, 12, and 50 after arrival of the calves to enumerate the number of fecal lactobacilli and coliforms. The number of fecal lactobacilli and coliforms were generally higher in experiment 3 than in experiment 4 (Table 5). There was no effect of either probiotic on the number of fecal lactobacilli. Coliform concentrations in the feces tended to be decreased by probiotic treatment. Treatment with CSPB reduced the average number of coliforms on $\mathrm{d} 5$ by 14 and $57 \%(P<0.05)$ in experiment 3 and 4 , respectively. Only in experiment 4, CSPB treatment significantly reduced the fecal coliform population on average. Treatment with MSPB did not affect coliforms in experiment 3 , but a slight, nonsignificant reduction was seen in experiment 4 on $\mathrm{d} 5$ and 12 .

\section{The Effect of Probiotics on Digestive and Respiratory Diseases}

The lowest percentage of animals that needed therapeutic treatment of digestive diseases and for all infec- tions combined was seen in experiment 1 (Table 6). Treatment with MSPB resulted in a lower percentage of animals being treated for digestive diseases and a lower number of treatments, but the effect was not statistically significant. In experiment 2 there was a high incidence of digestive and respiratory diseases. Treatment with MSPB did not elicit a clear influence on treatments for digestive or respiratory diseases.

The control animals in experiment 3 had a high incidence of gastrointestinal infections and a low incidence of respiratory diseases. Treatment with MSPB successfully reduced the percentage of animals suffering from digestive diseases and the number of treatments needed $(P<0.05)$. Overall, MSPB significantly reduced the percentage of animals in need of therapeutic treatment for any reason as well as the average amount of treatments needed.

The incidence of digestive diseases was moderate in experiment 4. Both MSPB and CSPB numerically lowered the percentage of animals treated and the average number of treatments needed. A strong inhibitory effect of probiotic treatment on therapeutic treatments for respiratory disease was found in experiment 4 . Both MSPB and CSPB significantly reduced the incidence of respiratory disease and its severity as expressed by the 


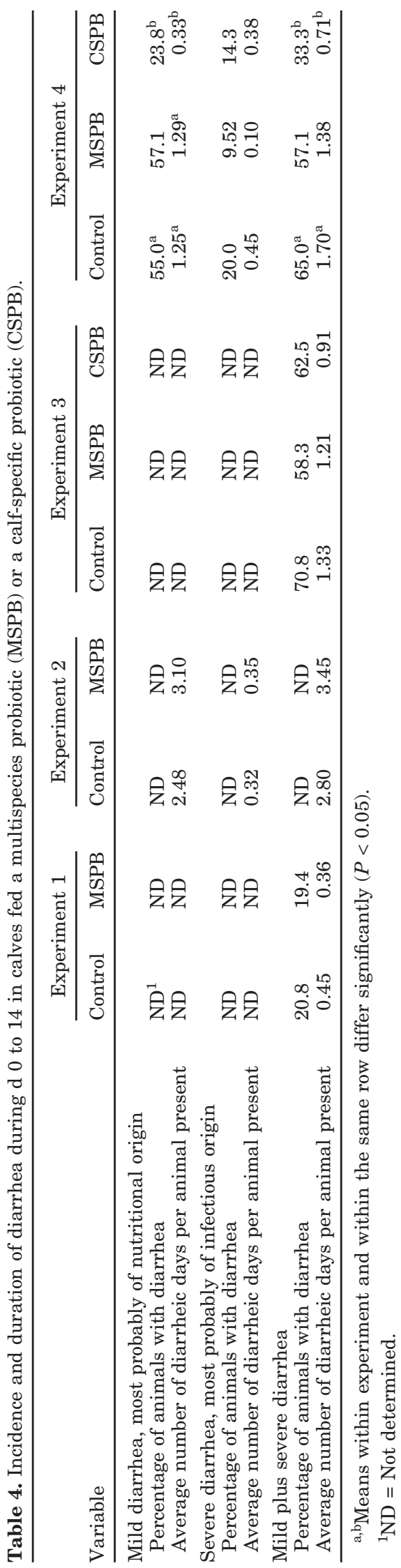

number of total treatments needed per affected animal (data not shown). Treatment with MSPB or CSPB significantly reduced the percentage of animals in need of therapeutic treatment of any cause and reduced the total number of treatments needed $(-72 \%$ and $-57 \%$, respectively).

\section{The Effect of Probiotic Treatment on GHS}

As described above, a GHS for the calves within the various treatments was calculated. A low GHS may be associated with high infection pressure that had caused diarrhea, respiratory disease, other infectious disease, and high mortality in the treatment group. Figure 1 shows that health was significantly compromised in experiment 4 compared with calves in the other experiments. In experiment 1 , the GHS was relatively high indicating a low infection pressure. Except in experiment 1, probiotics numerically raised the GHS. In experiment 2 and 3, treatment with either probiotic improved the GHS to a similar extent but the improvement was not statistically significant. Animal in experiment 4 showed the lowest GHS. In this situation, treatment with either probiotic resulted in a marked improvement of the GHS $(P<0.01)$. When all data were pooled, probiotic treatment was found to be associated with a significantly improved GHS.

It could be suggested that the stimulatory effect of probiotic treatment on growth depends on the baseline GHS. Indeed, the GHS of control calves and the MSPBinduced increase in ADG during wk 1 to 8 were negatively related when the data of the 4 experiments were pooled. Thus, the higher the GHS of control calves, the lower was the MSPB-induced increase in weight gain.

\section{DISCUSSION}

When a probiotic was added to the milk replacer of young veal calves there was an increase in BW gain during the first 2 wk after arrival. Similar observations were made by Cruywagen et al. (1996) who showed that administration of a monostrain probiotic ( $L$. acidophilus) resulted in maintenance of initial BW during wk 0 to 2 vs. a $1.6-\mathrm{kg}$ weight loss in nontreated animals. The growth-promoting effect of probiotics did not persist when treatment was continued. Possibly, the calves adapted to stressors such as transportation, new environment, change in diet, and infection pressure. Consequently, probiotic treatment may become less effective. This reasoning is supported by the observation that the probiotic-induced improvement in growth during the first 8 wk was negatively correlated with GHS. Thus, the positive effect of probiotics on growth performance of calves may only be present when their health status 
Table 5. Fecal coliform and Lactobacillus enumeration (log cfu/g \pm SD of feces) of calves fed a multispecies probiotic (MSPB) or a calfspecific probiotic (CSPB).

\begin{tabular}{|c|c|c|c|c|c|c|}
\hline Variable & \multicolumn{3}{|c|}{ Experiment 3} & \multicolumn{3}{|c|}{ Experiment 4} \\
\hline \multicolumn{7}{|c|}{ Fecal counts of coliforms $\left(\log _{10} \mathrm{cfu} / \mathrm{g}\right.$ of feces $)$} \\
\hline Day 12 & $8.14 \pm 0.85$ & $8.39 \pm 1.31$ & $7.68 \pm 0.93$ & $8.37^{\mathrm{a}} \pm 0.36$ & $7.75 \pm 0.71$ & $7.24^{\mathrm{b}} \pm 0.93$ \\
\hline Day 50 & $8.48 \pm 0.29$ & $8.19 \pm 0.80$ & $8.06 \pm 0.45$ & $5.97 \pm 2.24$ & $5.69 \pm 2.14$ & $5.01 \pm 2.44$ \\
\hline Average & $8.54 \pm 0.47$ & $8.48 \pm 0.67$ & $7.85 \pm 0.91$ & $6.99^{\mathrm{a}} \pm 0.90$ & $6.16^{\mathrm{c}} \pm 1.32$ & $4.99^{\mathrm{bd}} \pm 0.73$ \\
\hline Day 12 & $8.89 \pm 0.55$ & $9.05 \pm 0.53$ & $8.91 \pm 0.67$ & $8.03 \pm 0.83$ & $7.99 \pm 0.49$ & $7.85 \pm 0.45$ \\
\hline Day 50 & $8.00 \pm 0.69$ & $7.88 \pm 0.10$ & $8.21 \pm 0.34$ & $7.12 \pm 0.97$ & $7.07 \pm 0.76$ & $7.23 \pm 0.55$ \\
\hline Average & $8.82 \pm 0.49$ & $8.87 \pm 0.36$ & $8.82 \pm 0.30$ & $7.29 \pm 1.16$ & $7.31 \pm 0.70$ & $7.53 \pm 0.52$ \\
\hline
\end{tabular}

${ }^{\mathrm{a}, \mathrm{b}}$ Means within experiment and within the same row differ significantly $(P<0.05)$.

${ }^{c, d}$ Means within experiment and within the same row differ significantly $(0.05<P<0.10)$.

is compromised. Spanhaak et al. (1998) suggested that the health promoting effect of probiotic treatment is absent in healthy immunocompetent males. Between the experiments, there were marked differences in the GHS, growth rates, and probiotic-induced growth improvement. It is likely that combinations of origin of the calves, experimental conditions, and management systems influenced health, growth, and susceptibility to probiotics of the calves.

When results from the 4 experiments were pooled, there emerged a tendency that probiotic treatment resulted in reduced mortality. Further field trials with Belgian Blue calves with the same probiotic preparations confirmed this finding. In these experiments, there were 85 treated animals and 70 untreated animals with 0 and 4 deceased calves, respectively. The reduction in mortality using probiotics has been shown previously by different research groups in feeding experiments with piglets (Kyriakis et al., 1999), and hens (Yoruk et al., 2004), or in challenge models with Salmo- nella-exposed mice (Silva et al., 1999; Gill et al., 2001), and piglets infected with Escherichia coli (Underdahl, 1983).

In experiments 3 and 4 , it was found that probiotic treatment systemically reduced the occurrence of diarrhea. In experiment 4 , the CSPB preparation significantly depressed the incidence and duration of mild and total diarrhea. This outcome agrees with that of other studies in calves (Abe et al., 1995; Donovan et al., 2002; Khuntia and Chaudhary, 2002), but in other studies (Morrill et al., 1977; Cruywagen et al., 1996), no probiotic-induced reduction of the occurrence of diarrhea was observed. It is interesting to note that treatment with CSPB significantly reduced mild diarrhea, most probably of nutritional origin, whereas MSPB did not. However, MSPB significantly reduced therapeutic treatments against gastrointestinal disease. Perhaps the positive effect of MSPB against gastrointestinal disease is based on a different mechanism of action than that of CSPB.

Table 6. Therapeutic treatments during the 8-wk starter phase in the calves fed a multispecies probiotic (MSPB) or a calf-specific probiotic (CSPB).

\begin{tabular}{|c|c|c|c|c|c|c|c|c|c|c|}
\hline \multirow[b]{2}{*}{ Variable } & \multicolumn{2}{|c|}{ Experiment 1} & \multicolumn{2}{|c|}{ Experiment 2} & \multicolumn{3}{|c|}{ Experiment 3} & \multicolumn{3}{|c|}{ Experiment 4} \\
\hline & Control & MSPB & Control & MSPB & Control & MSPB & CSPB & Control & MSPB & CSPB \\
\hline \multicolumn{11}{|l|}{ Therapeutic treatments against digestive diseases } \\
\hline Average number of treatments per animal present & 0.11 & 0.03 & 0.52 & 0.67 & $1.58^{\mathrm{a}}$ & $1.13^{\mathrm{b}}$ & 1.29 & 0.20 & 0.10 & 0.05 \\
\hline \multicolumn{11}{|l|}{ Therapeutic treatments against respiratory diseases } \\
\hline Percentage of treated animals & 19.1 & 23.6 & 51.6 & 48.4 & 8.33 & 8.33 & 4.17 & $70.0^{\mathrm{ac}}$ & $23.8^{\mathrm{b}}$ & $42.9^{\mathrm{d}}$ \\
\hline Average number of treatments per animal present & 0.28 & 0.46 & 0.77 & 0.58 & 0.17 & 0.08 & 0.04 & $1.35^{\mathrm{a}}$ & $0.33^{\mathrm{b}}$ & $0.62^{\mathrm{b}}$ \\
\hline Percentage of treated animals & 24.7 & 25.0 & 64.5 & 71 & $75.0^{\mathrm{a}}$ & $45.8^{\mathrm{b}}$ & 62.5 & $75.0^{\mathrm{ac}}$ & $33.3^{\mathrm{b}}$ & $47.62^{\mathrm{d}}$ \\
\hline Average number of treatments per animal present & 0.52 & 0.63 & 1.35 & 1.26 & $1.75^{\mathrm{a}}$ & $1.25^{\mathrm{b}}$ & 1.38 & $1.55^{\mathrm{ac}}$ & $0.43^{\mathrm{b}}$ & $0.67^{\mathrm{d}}$ \\
\hline
\end{tabular}


All experiments were conducted during winter. To prevent excessive gastrointestinal and respiratory disease (typically associated with this season), animals were prophylactically treated with antibiotics. Use of any type of antibiotic is associated with the risk of developing antibiotic-associated diarrhea (BergogneBerezin, 2000). Probiotics are able to prevent or decrease the duration of this type of diarrhea (Siitonen et al., 1990; Vanderhoof et al., 1999). It could be hypothesized that the effects of probiotics are influenced by the use of antibiotics. Rollin et al. (1986) and Mero et al. (1985) concluded that use of selected antibiotics resulted in diarrhea and malabsorption in calves. In particular, treatment with neomycin resulted in diarrhea for all treated calves. However, the doses used were very high compared with doses used in experiments 2 and 4 ( 5 vs. $0.49 \mathrm{~g} / \mathrm{d})$. In contrast, similar low inclusion rates of neomycin and oxytetracycline in the diet of veal calves resulted in improved fecal scores compared with nontreated calves (Heinrichs et al., 2003). Whether the antibiotic treatments influenced the observed probiotic effects remains disputable. The growth-promoting effect during wk 1 to 2 is more likely a consequence of reducing weight-loss induced by stress factors during this adaptation period. However, the antidiarrheal effect might be affected by the antibiotics used.

In experiment 4 , with a high percentage of the control calves requiring therapeutic treatment against respiratory diseases, both MSPB and CSPB preparations diminished the need to treat respiratory infections. To our knowledge, the positive influence of oral probiotics on respiratory health in calves has not been reported previously. Hatakka et al. (2001) reported a reduction of antibiotic treatments against respiratory infections in children after long-term treatment with the probiotic Lactobacillus rhamnosus GG. That study, and a similar study published by Saavedra et al. (2004), showed that consumption of a probiotic resulted in a significant reduction of antibiotic use for various causes. The present results indicate that probiotics may reduce antibiotic use in veterinary practice as suggested by Conway and Wang (2000). Probiotics may not only reduce losses due to direct and indirect costs of respiratory disease, i.e., antibiotic usage and growth repression, respectively, but they might contribute to reducing microbial resistance against veterinary and human antibiotics.

It is possible that MSPB and CSPB have different mechanisms of action. The 2 probiotics have different origins and compositions. The CSPB preparation contains strains from a single genus, namely Lactobacillus. The MSPB preparation contains different species ( Lactobacillus, Lactococcus, and Enterococcus) and thereby combines certain health promoting properties that are species-specific. It could be suggested that CSPB colonizes the host more efficiently than MSPB because the former preparation contains calf-specific bacteria. Indeed, the number of fecal coliforms was lower when CSPB instead of MSPB was administered. Vinderola et al. (2004) performed a feeding experiment with mice fed different probiotic strains and tested whether the immunomodulating capacity of indigenous probiotics would differ from that of exogenous probiotic bacteria. It was found that indigenous strains were more effective at lower doses than exogenous bacteria, indicating that mucosal colonization is host specific. As mentioned, the evidence for calf-specific bacteria colonizing calves efficiently is not strong. It should be noted that we tested the effect of combinations of strains rather than individual strains. We have reviewed data pointing at synergistic effects resulting in the superiority of a multispecies probiotic compared with a multistrain probiotic (Timmerman et al., 2004). Such an effect would reduce any difference in the efficacy between MSPB and CSPB preparations used in this study.

\section{CONCLUSIONS}

It is clear from this study in 1-wk-old veal calves that administration of probiotics may increase BW gain during the first 2 wk of use. The probiotic-induced increase in weight gain for the period of 1 to 8 wk was greater when the calves were considered less healthy. Body weight and FE at 8 wk after arrival were significantly improved by probiotic treatment. Probiotic treatment reduced the incidence of diarrhea and the average number of diarrheic days. Mortality tended to be lower after feeding a milk replacer with probiotics.

Although there were differences in outcomes between experiments, it can be concluded that the supply of probiotics reduced the necessity of antibiotic treatments against digestive and respiratory diseases. Further experiments are required to study underlying mechanisms and to evaluate the potential of probiotics to improve respiratory health in the veal calf production.

\section{ACKNOWLEDGMENTS}

The research was supported partly by the Dutch Ministry of Economic Affairs (SENTER). The authors wish to thank Raymond Blankenstein and Cissy Warmerdam for technical assistance, Klaas Frankena for help with statistical analyses, and Cees Jansen and Mathieu Lam for taking care of the calves.

\section{REFERENCES}

Abe, F., N. Ishibashi, and S. Shimamura. 1995. Effect of administration of bifidobacteria and lactic acid bacteria to newborn calves and piglets. J. Dairy Sci. 78:2838-2846. 
Bergogne-Berezin, E. 2000. Treatment and prevention of antibiotic associated diarrhea. Int. J. Antimicrob. Agents 16:521-526.

Blecha, F., S. L. Boyles, and J. G. Riley. 1984. Shipping suppresses lymphocyte blastogenic responses in Angus and Brahman $\times$ Angus feeder calves. J. Anim. Sci. 59:576-583.

Conway, P. L., and X. Wang. 2000. Specifically targeted probiotics can reduce antibiotic usage in animal production. Asian-Australas. J. Anim. Sci. 13:358-361.

Cray, W. C., T. A. Casey, B. T. Bosworth, and M. A. Rasmussen. 1998. Effect of dietary stress on fecal shedding of Escherichia coli O157:H7 in calves. Appl. Environ. Microbiol. 64:1975-1979.

Cruywagen, C. W., I. Jordaan, and L. Venter. 1996. Effect of Lactobacillus acidophilus supplementation of milk replacer on preweaning performance of calves. J. Dairy Sci. 79:483-486.

Donovan, D. C., S. T. Franklin, C. C. Chase, and A. R. Hippen. 2002. Growth and health of Holstein calves fed milk replacers supplemented with antibiotics or Enteroguard. J. Dairy Sci. 85:947-950.

Fuller, R. 1989. Probiotics in man and animals. J. Appl. Bacteriol. 66:365-378.

Gill, H. S., Q. Shu, H. Lin, K. J. Rutherfurd, and M. L. Cross. 2001. Protection against translocating Salmonella typhimurium infection in mice by feeding the immuno-enhancing probiotic Lactobacillus rhamnosus strain HN001. Med. Microbiol. Immunol. 190:97-104.

Hartemink, R., V. R. Domenech, and F. M. Rombouts. 1997. LAMVAB-A new selective medium for the isolation of lactobacilli from faeces. J. Microbiol. Methods 29:77-84.

Hatakka, K., E. Savilahti, A. Ponka, J. H. Meurman, T. Poussa, L. Nase, M. Saxelin, and R. Korpela. 2001. Effect of long term consumption of probiotic milk on infections in children attending day care centres: Double blind, randomised trial. BMJ 322:1-5.

Heinrichs, A. J., C. M. Jones, and B. S. Heinrichs. 2003. Effects of mannan oligosaccharide or antibiotics in neonatal diets on health and growth of dairy calves. J. Dairy Sci. 86:4064-4069.

Khuntia, A., and L. C. Chaudhary. 2002. Performance of male crossbred calves as influenced by substitution of grain by wheat bran and the addition of lactic acid bacteria to diet. Asian-Australas. J. Anim. Sci. 15:188-194.

Kyriakis, S. C., V. K. Tsiloyiannis, J. Vlemmas, K. Sarris, A. C. Tsinas, C. Alexopoulos, and L. Jansegers. 1999. The effect of probiotic LSP 122 on the control of post-weaning diarrhoea syndrome of piglets. Res. Vet. Sci. 67:223-228.

Loerch, S. C., and F. L. Fluharty. 1999. Physiological changes and digestive capabilities of newly received feedlot cattle. J. Anim. Sci. 77:1113-1119.

McCullagh, A., and J. A. Nelder. 1989. Generalized Linear Models. 2nd ed. Chapman and Hall, London, UK.

Mero, K. N., R. E. Rollin, and R. W. Phillips. 1985. Malabsorption due to selected oral antibiotics. Vet. Clin. North Am. Food Anim. Pract. 1:581-588

Morrill, J. L., A. D. Dayton, and R. Mickelsen. 1977. Cultured milk and antibiotics for young calves. J. Dairy Sci. 60:1105-1109.
Nabuurs, M. J., G. J. van Essen, P. Nabuurs, T. A. Niewold, and J. van der Meulen. 2001. Thirty minutes transport causes small intestinal acidosis in pigs. Res. Vet. Sci. 70:123-127.

Postema, H. J., P. Franken, and J. B. van der Ven. 1987. A study in veal calves for a possible correlation between serum immunoglobulin levels, nutrition levels and risk of disease in the first few weeks of the fattening period. Tijdschr. Diergeneeskd. 112:665-671.

Rollin, R. E., K. N. Mero, P. B. Kozisek, and R. W. Phillips. 1986. Diarrhea and malabsorption in calves associated with therapeutic doses of antibiotics: Absorptive and clinical changes. Am. J. Vet. Res. 47:987-991.

Saavedra, J. M., A. Abi-Hanna, N. Moore, and R. H. Yolken. 2004. Long-term consumption of infant formulas containing live probiotic bacteria: Tolerance and safety. Am. J. Clin. Nutr. 79:261-267.

SAS Institute. 2000. SAS User's Guide, Statistics, version 8, vol. 1, 2, and 3. SAS Inst., Inc., Cary, NC.

Sheridan, J. F., C. Dobbs, D. Brown, and B. Zwilling. 1994. Psychoneuroimmunology: Stress effects on pathogenesis and immunity during infection. Clin. Microbiol. Rev. 7:200-212.

Siitonen, S., H. Vapaatalo, S. Salminen, A. Gordin, M. Saxelin, R. Wikberg, and A. L. Kirkkola. 1990. Effect of Lactobacillus GG yoghurt in prevention of antibiotic-associated diarrhoea. Ann. Med. 22:57-59.

Silva, A. M., E. A. Bambirra, A. L. Oliveira, P. P. Souza, D. A. Gomes, E. C. Vieira, and J. R. Nicoli. 1999. Protective effect of bifidus milk on the experimental infection with Salmonella enteritidis subsp typhimurium in conventional and gnotobiotic mice. J. Appl. Microbiol. 86:331-336.

Soderholm, J. D., and M. H. Perdue. 2001. Stress and the gastrointestinal tract II. Stress and intestinal barrier function. Am. J. Physiol. Gastrointest. Liver Physiol. 280:G7-G13.

Spanhaak, S., R. Havenaar, and G. Schaafsma. 1998. The effect of consumption of milk fermented by Lactobacillus casei strain Shirota on the intestinal microflora and immune parameters in humans. Eur. J. Clin. Nutr. 52:899-907.

Timmerman, H. M., C. J. M. Koning, L. Mulder, F. M. Rombouts, and A. C. Beynen. 2004. Monostrain, multistrain and multispecies probiotics-A comparison of functionality and efficacy. Int. J. Food Microbiol. 96:219-233.

Underdahl, N. R. 1983. The effect of feeding Streptococcus faecium upon Escherichia coli induced diarrhea in gnotobiotic pigs. Prog. Food Nutr. Sci. 7:5-12.

Vanderhoof, J. A., D. B. Whitney, D. L. Antonson, T. L. Hanner, J. V. Lupo, and R. J. Young. 1999. Lactobacillus GG in the prevention of antibiotic-associated diarrhea in children. J. Pediatr. 135:564568.

Vinderola, C. G., M. Medici, and G. Perdigon. 2004. Relationship between interaction sites in the gut, hydrophobicity, mucosal immunomodulating capacities and cell wall protein profiles in indigenous and exogenous bacteria. J. Appl. Microbiol. 96:230-243.

Yoruk, M. A., M. Gul, A. Hayirli, and M. Macit. 2004. The effects of supplementation of humate and probiotic on egg production and quality parameters during the late laying period in hens. Poult. Sci. 83:84-88. 